\title{
B Acute Lymphoblastic Leukemia with Intrachromosomal Amplification of Chromosome 21
}

National Cancer Institute

\section{Source}

National Cancer Institute. B Acute Lymphoblastic Leukemia with Intrachromosomal

Amplification of Chromosome 21. NCI Thesaurus. Code C130040.

B acute lymphoblastic leukemia characterized by amplification of a portion of chromosome 21. It usually occurs in children and is associated with an adverse prognosis. 